\section{Tecnologías para la detección de ocupación en edificios} \section{Dafni Mora ${ }^{1,3}$ (iD, Marilena De Simone ${ }^{2}$ (D), Miguel} Chen Austin 1,3 (iD)

1 Universidad Tecnológica de Panamá, Facultad de Ingeniería Mecánica, 2 Department of Environmental and Chemical Engineering, University of Calabria, Italia, 3 Grupo de Investigación en Energética y Confort en Edificaciones Bioclimáticas (ECEB), Universidad Tecnológica de Panamá, Facultad de Ingeniería Mecánica

dafni.mora@utp.ac.pa,marilena.desimone@unical.it, miguel.chen@utp.ac.pa DOI 10.33412/pri.v11.1.2530

\section{(잉(-)}

Resumen: Una importante parte del consumo de energía de los edificios está relacionado con el comportamiento de los ocupantes. Lo que significa que es necesario conocer con mayor precisión cómo se comporta y se mueve el usuario dentro de los ambientes, y este es uno de los retos que requiere conocer los tipos de tecnologías para la detección de la ocupación en edificios. En este artículo presentamos un resumen de las tecnologías utilizadas en la actualidad para el control de la ocupación en edificios, de manera de tener un marco de referencia y además poder indagar hacia donde se pueden dirigir las investigaciones futuras, tomando en cuenta el desarrollo actual.

Palabras claves: ocupación del edificio, monitoreo, rendimiento del edificio, sensores

Title: Technologies for occupancy monitoring in buildings

Abstract: An essential part of the energy consumption of buildings is related to the occupant's behavior. Which means that it is necessary to know more precisely how the user behaves and moves within the environments, and this is one of the challenges that require knowing the technology for occupancy monitoring in buildings. This article presents a summary of the technologies currently used for the control of building occupancy with the aim of investigating where potential future research can be directed, considering the current development.

Keywords: building occupancy, monitoring, building performance, sensors.

Tipo de artículo: original

Fecha de recepción: 15 de octubre de 2019

Fecha de aceptación: 22 de enero de 2020

\section{Introducción}

Una de las barreras más importantes cuando se busca mejorar la eficiencia energética de los edificios, es la falta de conocimiento sobre los factores que determinan el uso final de la energía.

Actualmente, con la implementación de nuevas tecnologías orientadas al ahorro energético y a la certificación medioambiental de edificios, tales como la certificación LEED [1] y Green Globes [2], surge un nuevo enfoque, relacionado a como incide el comportamiento del ocupante en el uso de la energía [3].

A menudo, se reporta una discrepancia significativa entre las predicciones o estimaciones y el uso real de la energía total en los edificios. Las razones de esta diferencia son pocos conocidos y en gran parte tiene que ver más con la conducta humana que con el diseño del edificio, de acuerdo a expertos del Programa de Energía en Edificios y Comunidades de la Agencia Internacional de Energía (IEA-EBC), y resultados del proyecto internacional Anexo 66: Definición y simulación del Comportamiento de los Ocupantes en Edificios [4].

En cuanto a edificios de similares características constructivas y operativas (tamaño, número de ocupantes, electrodomésticos, orientación, ventanas, cortinas, etc.) se reporta una variación considerable en el consumo de energía entre ellos, que ha llevado a considerar, que más allá de las nuevas tecnologías de ahorro de energía y/o las aplicaciones de energías renovables que se utilicen, es necesario un enfoque relativo al comportamiento del ocupante en relación con el consumo de energía del edificio [5][6].

En el contexto de estas investigaciones, el comportamiento del ocupante, se puede definir, como el uso del espacio, sistemas y otros servicios dentro de la casa que pueden influir en el consumo de energía para la climatización del ambiente y producción de agua caliente [7]. Además, se puede precisar, como las acciones observables o reacciones de una persona en respuesta a estímulos externos 0 internos, 0 acciones 0 reacciones de una persona para adaptarse a las condiciones ambientales como la temperatura o la calidad del aire interior [8].

El consumo de energía relacionado con el comportamiento de los ocupantes en los edificios, por ejemplo el ajuste del termostato para la comodidad, el encender y apagar las luces, apertura y cierre de las ventanas, apertura y cierre de las persianas de las ventanas, y el movimiento entre los ambientes internos, es un tema clave para la optimización del diseño de los edificios, el diagnóstico de la energía, la evaluación del desempeño, y de la simulación energética, debido a su impacto significativo en el consumo de energía real y en la calidad del ambiente interior [4].

El uso de energía en edificios es altamente dependiente de las actividades de los residentes, el monitoreo de la ocupación y el modelado son importante para facilitar el uso eficiente de la energía y el manejo del confort. Uno de los principales retos en la determinación de las actividades de los ocupantes es la construcción de las redes de sensores de ocupación en los edificios. Según los autores [9], los factores involucrados en el consumo total de energía de los edificios se pueden clasificar en siete categorías:

a. Condiciones climáticas (ej. temperatura exterior, radiación solar y velocidad del viento),

Prisma Tecnológico | Vol. II, n. ${ }^{\circ}$ I, edición 2020. 
b. Características relacionadas con el edificio (ej. Área y orientación),

c. Características del usuario, excepto factores sociales y económicos,

d. Sistemas y operación presentes en el edificio (suministro de agua caliente y ambientes climatizados),

e. Comportamientos del ocupante y actividades,

f. Factores sociales y económicos (ej. costo de energía y nivel educativo del ocupante),

g. Calidad del aire interior requerida.

Un edificio inteligente es capaz de gestionar su ambiente interior a través de técnicas informáticas para optimizar la eficiencia energética, el bienestar de los ocupantes, la seguridad y la productividad. Su objetivo es conseguir tanto el ahorro energético, como la satisfacción del ocupante a través de servicios al usuario [10].

El objetivo de este artículo es describir las técnicas existentes para el control y monitoreo de la ocupación en los edificios, con el fin de tener un marco de referencia de los aspectos a considerar para la selección de alguna tecnología específica, dependiendo de la aplicación deseada. También, este estudio permitirá indagar hacia donde se pueden dirigir las investigaciones futuras, tomando en cuenta el desarrollo actual de las diferentes tecnologías.

\section{Enfoques para el monitoreo de la ocupación}

La detección de la ocupación es definida como la detección en tiempo real de la presencia en un ambiente, puede tener dos valores: ocupado y desocupado. Los autores en [11] dividen el nivel de detalle de la información de ocupación que podemos obtener en diferentes niveles o tipos de resolución como se presenta en la figura 1.

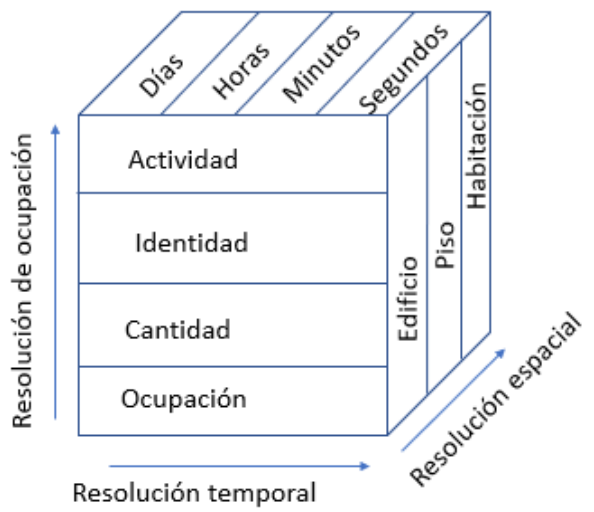

Figura 1. Niveles de resolución de ocupación [11].

Dependiendo del tipo de resolución requerido para cada aplicación, se deben seleccionar los sensores a utilizar. Otros aspectos que se deben tomar en cuenta son los costos (individuales de software y hardware, integración con los sistemas existentes y mantenimiento), tipos de fuentes de poder, almacenamiento de los datos, rango de monitoreo, precisión y fallos. De igual manera los autores en [12] proponen un marco de categorización, que clasifica los sistemas de detección de ocupación en nueve categorías, como se muestra en la tabla 1.

Dicha tabla permite agrupar y asignar un parámetro de medición de carácter cuantitativo o cualitativo, dependiendo del detalle y especificaciones del estudio que se quiera realizar con respecto a la ocupación. Los parámetros fueron definidos en base a trabajos anteriores sobre el comportamiento de la ocupación, buscando palabras claves en artículos de revistas y conferencias.

Tabla 1. Categorización de los sistemas de detección de ocupación [12]

\begin{tabular}{|l|l|l|}
\hline $\mathrm{n}^{\circ}$ & \multicolumn{1}{|c|}{ Categoría } & \multicolumn{1}{c|}{ Parámetro de medición } \\
\hline 1 & $\begin{array}{l}\text { Tipo de información de } \\
\text { ocupación }\end{array}$ & Presencia, actividad y comportamiento. \\
\hline 2 & $\begin{array}{l}\text { Relación entre el } \\
\text { sistema y el ocupante }\end{array}$ & Anónimo, individual o multitud. \\
\hline 3 & Estrategia de monitoreo & $\begin{array}{l}\text { "augment objects", "augment persons", } \\
\text { "augment the environment", reutilizar } \\
\text { infraestructura o la interacción con el } \\
\text { ocupante. }\end{array}$ \\
\hline 4 & Granularidad espacial: & Lugar, edificio, piso, espacio, objeto. \\
\hline 5 & Granularidad temporal: & Periódico, basado en un evento especifico. \\
\hline 6 & Cobertura espacial & Sitio, edificio, espacio, objeto. \\
\hline 7 & Cobertura temporal & Pasado, presente, futuro. \\
\hline 8 & Modalidad del sensor & $\begin{array}{l}\text { Datos ocupantes, fuerza, luz visible, luz } \\
\text { infrarroja, sonido, ultrasonido, aire, } \\
\text { radiación electromagnética, electricidad, } \\
\text { agua, datos de sistemas de calefacción, } \\
\text { refrigeración y ventilación. }\end{array}$ \\
\hline 9 & Métodos y Modelos & $\begin{array}{l}\text { Reglas condicionales, modelos basados en } \\
\text { agentes, estocásticos, machine learning, } \\
\text { algoritmos de predicción, análisis de } \\
\text { señales, heurísticos. }\end{array}$ \\
\hline
\end{tabular}

El problema de determinar la localización del usuario en un edificio (incluyendo información sobre cuando entra o sale de la habitación) es conocido como el problema de detección de la ocupación. Es importante conocer quien está en un espacio en un momento especifico para poder determinar las operaciones de dispositivos relacionados con el uso de la energía para una mayor comodidad.

Dependiendo del tipo de sistema de medición de la ocupación utilizado, se puede definir cuál información relativa a la presencia de los ocupantes y su movimiento se puede obtener. La información de la ocupación se puede clasificar en tres categorías observables: Propiedades espacio-temporal, propiedades del comportamiento y propiedades fisiológicas (ver Fig. 2).

Las propiedades espacio-temporal proporcionan la información integral de la ocupación a nivel detallado. Estas propiedades son las que tienen relación directa con el consumo energético en los edificios.

Los métodos utilizados para la detección de la ocupación se pueden clasificar en tres categorías [13]:

Método de detección directa, se refiere al despliegue de sensores 0 redes de sensores para detectar directamente la presencia 0 el seguimiento de los ocupantes a través de los espacios.

Método de modelado, utiliza métodos de análisis de datos estadísticos en el modelado de la ocupación. 
Método combinado, es una combinación de métodos de análisis avanzados, como los métodos estadísticos, con medidas físicas. Los autores en [14] propusieron otra clasificación de los sistemas de detección de la ocupación, de acuerdo a las propiedades espacio-temporal y al tipo de sensor utilizado. Está basada en tres aspectos:

Método, si necesita un terminal (ej. Celular, identificador de radio frecuencia (RFID) u otro) [16].

Función, en individualizado o no-individualizado, si logra o no reconocer el usuario presente. Están siendo utilizados en investigaciones de confort térmico personalizado [17].
Infraestructura, en implícito o explícito, si el propósito inicial es medir la ocupación o es su función secundaria. Los autores Melfi et. al [18], realizaron la medición de la ocupación del edificio mediante el uso de la infraestructura de red existente.

En la tabla 2, diferentes sensores fueron clasificados en base a los tres aspectos anteriores (método, función e infraestructura), mientras que en la tabla 3 estos sensores fueron clasificados dependiendo de cuál de las propiedades Espacio-temporal logra identificar. De esta manera, todos los tipos de sensores mostrados permiten la localización del ocupante y permite la identificación de presencia y ausencia en el sitio.

Tabla 2. Clasificación de los sistemas de detección de ocupación [14].

\begin{tabular}{|c|c|c|c|c|c|c|c|c|c|c|}
\hline & & \multicolumn{9}{|c|}{ Sensores } \\
\hline & & $\mathrm{CO}_{2}$ & PIR & Ultrasonido & Imagen & Sonido & $\begin{array}{c}\text { Señales } \\
\text { EM }\end{array}$ & $\begin{array}{l}\text { Medidor } \\
\text { corriente }\end{array}$ & $\begin{array}{l}\text { Aplicaciones } \\
\text { (App.) }\end{array}$ & $\begin{array}{l}\text { Fusión de } \\
\text { sensores }\end{array}$ \\
\hline \multirow[t]{2}{*}{ Método } & Terminal & & & & & & $x$ & & & $x$ \\
\hline & Sin terminal & $x$ & $x$ & $x$ & $x$ & $x$ & & $x$ & $x$ & $x$ \\
\hline \multirow[t]{2}{*}{ Función } & Individualizado & & & & & & & & & $x$ \\
\hline & No-Individualizado & $x$ & $x$ & $\mathrm{x}$ & $x$ & $x$ & $x$ & $x$ & $x$ & $x$ \\
\hline \multirow[t]{2}{*}{ Infraestructura } & Implícito & & & & $x$ & & $x$ & & $x$ & $x$ \\
\hline & Explícito & $x$ & $x$ & $x$ & $x$ & $x$ & $x$ & $x$ & & $x$ \\
\hline
\end{tabular}

Tabla 3. Clasificación de los sistemas de detección de ocupación en base a las propiedades espacio-temporales [14].

\begin{tabular}{|l|c|c|c|c|c|c|c|c|c|}
\hline & \multicolumn{9}{c|}{ Sensores } \\
\hline & $\mathrm{CO}_{2}$ & $\mathrm{PIR}$ & Ultrasonido & Imagen & Sonido & Señales EM & Medidor corriente & Aplicaciones & Fusión de sensores \\
\hline Localización & $\mathrm{x}$ & $\mathrm{x}$ & $\mathrm{x}$ & $\mathrm{x}$ & $\mathrm{x}$ & $\mathrm{x}$ & $\mathrm{x}$ & $\mathrm{x}$ & $\mathrm{x}$ \\
\hline Presencia & $\mathrm{x}$ & $\mathrm{x}$ & $\mathrm{x}$ & $\mathrm{x}$ & $\mathrm{x}$ & $\mathrm{x}$ & $\mathrm{x}$ & $\mathrm{x}$ & $\mathrm{x}$ \\
\hline Cantidad & $\mathrm{x}$ & & & $\mathrm{x}$ & & $\mathrm{x}$ & & $\mathrm{x}$ & $\mathrm{x}$ \\
\hline Actividad & $\mathrm{x}$ & & & $\mathrm{x}$ & & $\mathrm{x}$ & $\mathrm{x}$ & $\mathrm{x}$ & $\mathrm{x}$ \\
\hline Identidad & & & & $\mathrm{x}$ & & $\mathrm{x}$ & & $\mathrm{x}$ & \\
\hline Seguimiento & & & & $\mathrm{x}$ & & $\mathrm{x}$ & & $\mathrm{x}$ \\
\hline
\end{tabular}

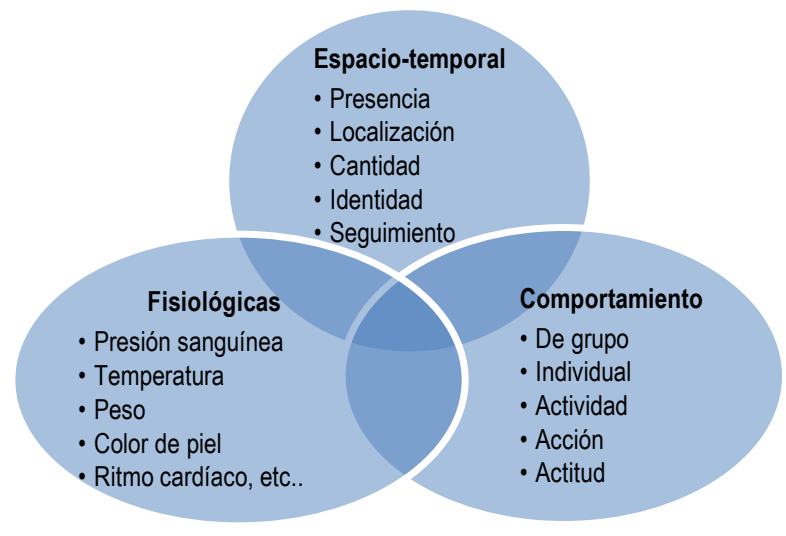

Figura 2. Propiedades espacio-temporal, fisiológicas y de comportamiento de medida de ocupación [15].

\section{Tecnologías de detección de ocupación}

Con la tendencia mundial orientada a edificios de bajo consumo energético [19] [20], los controles de ocupación están siendo utilizados para reducir el uso de la energía en los edificios, tales como para el control del aire acondicionado, ventilación e iluminación. Algunos dispositivos son controlados por sensores de ocupación y realizan de forma independiente la toma de decisiones relacionadas al comportamiento de los ocupantes. Por ejemplo un típico $30 \%$ de ahorro de energía se puede lograr con sensores de ocupación para controlar la iluminación [21].

Las tecnologías para medir la ocupación pueden ser divididas en seis categorías: basadas en imágenes, contacto y mecánico, detección de movimiento, señal electromagnética, parámetros ambientales, registro manual de datos y detección de consumo [22] (ver Fig. 3). Los sensores funcionan en base a los fundamentos físicos generales y aún presentan deficiencias, tales como la necesidad de desarrollar elementos de detección avanzados que puedan capturar a los ocupantes en una zona térmica con precisión, además que la mayoría de los sensores dependen de una fuente de alimentación externa para un experimento a largo plazo, creando desafíos de costos y cableado para la implementación a gran escala. De igual manera el consumo de energía del conjunto integrado: unidad de procesamiento, comunicación y el elemento sensor en sí.

Las tecnologías basadas en imágenes, detectan la información electromagnética y la transmiten en forma de matriz. Consisten en cámaras de luz visible, cámaras de luminancia, cámaras infrarrojas (IR) o térmicas, sensores de nivel de luz y cámaras estereoscópica. No están basados en terminales y pueden utilizarse para proporcionar función individualizada [14]. Hay altas 
precisiones de detección con sensores de cámara de buena calidad. Han demostrado proporcionar información de la ocupación en una amplia gama de su resolución (identidad, cantidad, actividad y otros) [23], sin embargo, pueden tener altos costos de instalación y mantenimiento y presentan problemas de privacidad

De contacto y mecánico, ambos detectan el cambio en el estado de un componente del edificio con el que los ocupantes interactúan con frecuencia, como una puerta, una ventana o el aire acondicionado. Los ejemplos de esta categoría son interruptores de lámina (Reed contact) [24], placas de puertas (door badges), los tapetes piezoeléctricos, los rayos infrarrojos (IR beams), tarjetas de acceso y bobinas magnéticas.

Sensores de movimiento, detectan la presencia o ausencia de un ocupante a través de sus movimientos. El más común es el sensor infrarrojo pasivo (PIR). Todos los objetos, incluidos los seres humanos con una temperatura por encima del cero absoluto, emiten energía térmica en forma de radiación. La radiación infrarroja emitida es invisible para el ojo humano, pero puede ser detectada por dispositivos como los sensores PIR [14]. Otros tipos son los Doppler ultrasónico, Doppler de microondas y rango ultrasónico.

Señal electromagnética, las señales relevantes para la detección de ocupación incluyen: fidelidad inalámbrica (WIFI), Bluetooth, banda ultra ancha (UWB), etiquetas de identificación por radiofrecuencia (RFID) y el sistema de posicionamiento global (GPS). El sistema generalmente consiste en un nodo de transmisión y otro de recepción. La señal transmitida puede consistir en una serie corta de pulsos o señales de radio modulada [14]. Es importante tener en cuenta que las señales de alta frecuencia que se transmiten a través del aire se ven afectadas por la humedad, la presencia de otras señales y muchos otros factores ambientales.

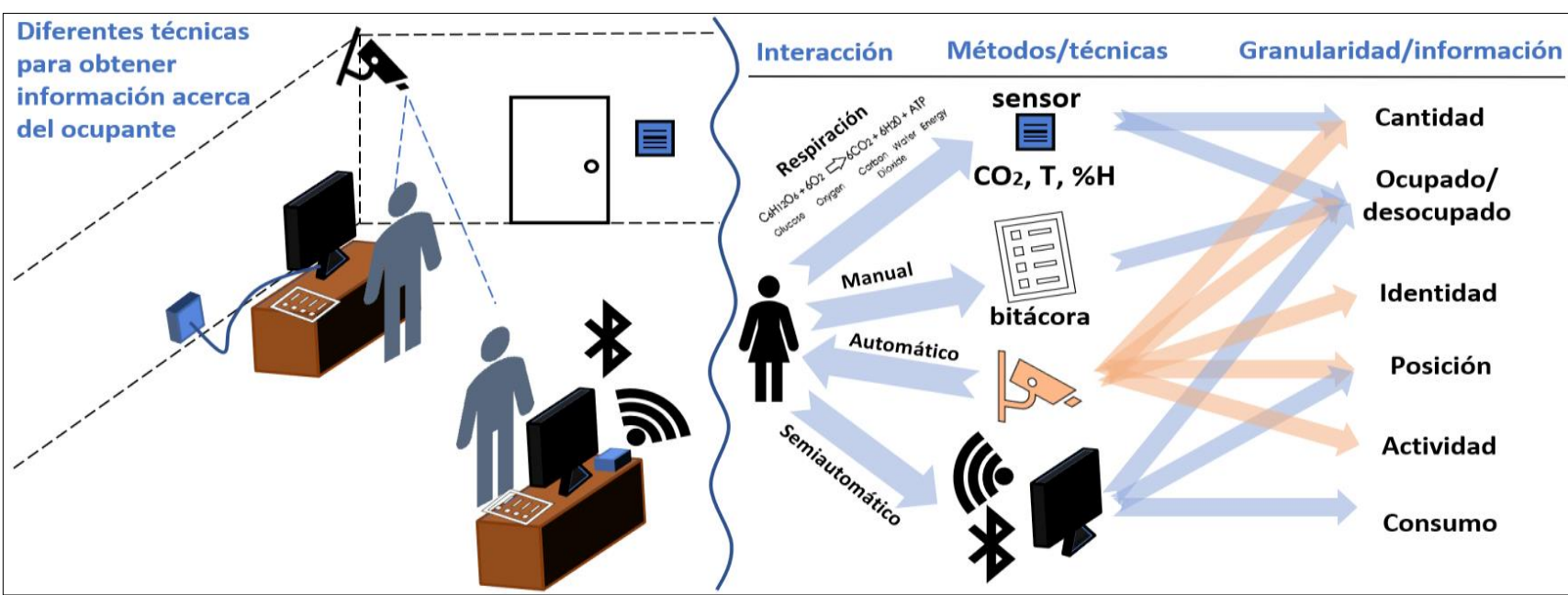

Figura 3. Diferentes tecnologías o técnicas para obtención de información del ocupante.

Parámetros ambientales, son un conjunto de diferentes sensores, siendo los más efectivos para la detección de la ocupación: Dióxido de carbono $\left(\mathrm{CO}_{2}\right)$, humedad relativa, temperatura, compuestos orgánicos volátiles (VOC), acústicos. Los que se consideran más efectivos para detectar presencia son los acústicos y de niveles de $\mathrm{CO}_{2}$ [24-26]. Los acústicos cubren la captura de sonido soportable mediante los micrófonos y configuraciones de transmisión para estimar la proximidad [12]. Con estos sensores es posible obtener mapas de confort.

Mediante el registro manual de datos, la metodología más básica y directa utilizada para la recopilación de información de ocupación es la aplicación de cuestionarios y entrevistas [27,28]. Los métodos en esta categoría son la observación y registro manual, datos de ocupantes basados en internet y las interacciones con dispositivos [18].

Detección de consumo, cubre la medición del consumo de electricidad y de agua. El cambio en el consumo de energía de un dispositivo cuando cambia de un estado inactivo a activo proporciona información con la cual se puede inferir la ubicación y presencia de los usuarios [14].
Otra modalidad ampliamente utilizada en la actualidad es la "fusión de sensores", con esta técnica se busca maximizar las ventajas de cada uno de los sensores y lograr una combinación que permita mayor precisión en la detección de la ocupación y superar las limitaciones individuales de cada uno de los sensores $[14,24,29]$.

\subsection{Parámetros a considerar al seleccionar un sensor}

En las últimas décadas, las investigaciones del comportamiento de los ocupantes en los espacios acondicionados han sido realizadas utilizando alguna de las tecnologías descritas en este artículo, de manera individual, sin embargo, actualmente con los avances de la tecnología orientadas hacia el internet de las cosas (loT), es posible la implementación de diferentes sensores y obtener mejores resultados, que nos permitan definir modelos más precisos para estimar, tanto la presencia, como la interacción de los ocupantes con los diferentes sistemas de los edificios. De acuerdo con las tecnologías descritas, se pueden identificar algunos parámetros de rendimiento, que permitan evaluar las opciones al momento de 
requerir la implementación de alguna de estos sistemas, tales como: costo, área de implementación, tipo de recolección de datos, problemas de privacidad, tipo de fuente de poder, rango de detección, precisión de la detección, almacenamiento de datos y limitaciones asociadas a cada sensor [22].

\subsection{Retos al seleccionar un sensor}

En este tema, existen retos importantes aún por afrontar en lo que corresponde a la estimación y detección de la ocupación, tales como el estudio de la mejor ubicación de los sensores de manera de optimizar su desempeño, así como el aumento de técnicas que incluyan la fusión de sensores, a manera de poder compensar las limitaciones y mejorar el rendimiento final de las tecnologías individuales y los algoritmos desarrollados para estimar y detectar la ocupación sin necesidad de la intervención humana [30]. Existen desafíos claros en términos del elemento sensor, del consumo de energía, del procesamiento de los datos y en términos de comunicación, tales como investigar mecanismos de bajo consumo de energía para que los sensores puedan ser autónomos por largos periodos de tiempo. Otro aspecto por considerar es la comunicación que determina la frecuencia con la que los datos se almacenan y que consume la mayor parte de energía de todo el sensor y como se puede configurar una red de comunicación para minimizar el consumo total de energía.

En las últimas décadas, el monitoreo de la ocupación se ha enfocado en la utilización de un solo sensor, sin embargo, con el desarrollo del internet de las cosas (loT), la detección de ocupantes y la adquisición de datos no se limitan a un solo nodo.

\section{Discusión}

Las tecnologías presentadas anteriormente son utilizadas en la actualidad para el control de la ocupación en edificios y su implementación contribuye a disminuir el consumo de energía, costos de operación, así como a mejorar del rendimiento general de los edificios, dado que la información recopilada permite anticipar comportamientos y preferencias de los ocupantes.

Uno de los principales parámetros a considerar para la implementación de alguna de estas tecnologías, es el costo del equipo, el cual incluye los costos individuales de hardware y software, de instalación e integración con los sistemas e infraestructuras existentes, gastos de operación, mantenimiento y actualizaciones de los softwares. Otro aspecto importante, es la privacidad de los ocupantes, por lo cual debe siempre tenerse en cuenta su consentimiento y buscar un equilibrio entre el nivel de monitoreo y la privacidad de estos. Los demás aspectos se deben tener en cuenta, dependiendo de los resultados deseados.

\section{Conclusión}

En este artículo se realizó una revisión de las técnicas existentes para el control de la ocupación de edificios, de acuerdo al método, su función y la infraestructura vs el tipo de sensor utilizado. Adicionalmente se realizó una clasificación general de los sistemas de ocupación en bases a sus propiedades espacio temporal, siendo estas: localización, presencia, cantidad, actividad, identidad y seguimiento.

\section{Agradecimiento}

El investigador es apoyado por el Sistema Nacional de Investigación (SNI) de la Secretaria Nacional de Ciencia, Tecnología e Innovación de Panamá (SENACYT).

\section{Referencias}

[1] U.S. Green Building Council, LEED 2009 for new construction and major renovations, 2009.

[2] (2009) Green Globes, The green building initiative. [Online] Disponible en: http://www.greenglobes.com/home.asp.

[3] D. Mora, C. Carpino, M. De Simone, Energy consumption of residential buildings and occupancy profiles. A case study in Mediterranean climatic conditions., Energy Efficiency. 2017. doi:10.1007/s12053-0179553-0.

[4] (2015) IEA, IEA-EBC Annex 66: Definition and simulation of occupant behavior in buildings. [Online] Disponible en: www.annex66.org.

[5] V. Fabi, R.V. Andersen, S. Corgnati, B.W. Olesen, Occupants' window opening behaviour: a literature review of factors influencing occupant behaviour and models, Building and Environment. 2012. vol. 58, 188198. doi:10.1016/j.buildenv.2012.07.009.

[6] A.F. Emery, C.J. Kippenhan, A long term study of residential home heating consumption and the effect of occupant behavior on homes in the Pacific Northwest constructed according to improved thermal standards, Energy. 2006, vol. 31, 677-693. doi:10.1016/j.energy.2005.04.006

[7] O. Guerra-Santin, Actual energy consumption in dwellings -the effect of energy performance regulations and occupant behaviour, IOS Press under the imprint Delft University Press, 2010.

[8] S. Chen, W. Yang, H. Yoshino, M.D. Levine, K. Newhouse, A. Hinge, Definition of occupant behavior in residential buildings and its application to behavior analysis in case studies, Energy and Buildings. 2015. vol. 104, 1-13. doi:10.1016/j.enbuild.2015.06.075.

[9] Z. Yu, B.C.M. Fung, F. Haghighat, H. Yoshino, E. Morofsky, A systematic procedure to study the influence of occupant behavior on building energy consumption, Energy and Buildings. 2011. vol. 43, 1409-1417.

[10] R. Yang, L. Wang, Development of multi-agent system for building energy and comfort management based on occupant behaviors, Energy and Buildings. 2013. vol. 53, 1-7. doi:10.1016/j.enbuild.2012.10.025.

[11] K. Christensen, R. Melfi, B. Nordman, B. Rosenblum, R. Viera, Using existing network infrastructure to estimate building occupancy and control plugged-in devices in user workspaces, International Journal Communication Networks and Distributed Systems. 2014. vol. 12, 429.

[12] M.B. Kjærgaard, F.C. Sangogboye, Categorization framework and survey of occupancy sensing systems, Pervasive and Mobile Computing. 38 (2017) 1-13. doi:10.1016/j.pmcj.2016.09.019.

[13] R. Zhang, K.P. Lam, Y.-S. Chiou, B. Dong, Information-theoretic environment features selection for occupancy detection in open office spaces, Building Simulation. 2012. vol. 5, 179-188. doi:10.1007/s12273-012-0075-6.

[14] T. Labeodan, W. Zeiler, G. Boxem, Y. Zhao, Occupancy measurement in commercial office buildings for demand-driven control applications A survey and detection system evaluation, Energy and Buildings. 93 (2015) 303-314. doi:10.1016/j.enbuild.2015.02.028.

[15] T. Teixeira, G. Dublon, A. Savvides, A survey of human-sensing: methods for detecting presence, count, location, track, and identity, ACM Computing Surveys. 2010. vol. 5, 1-35. http://www.eng.yale.edu/enalab/publications/human_sensing_enalab WIP.pdf.

[16] C. Martani, D. Lee, P. Robinson, R. Britter, C. Ratti, ENERNET: Studying the dynamic relationship between building occupancy and energy consumption, Energy and Buildings. 2012. vol. 47, 584-591. doi:10.1016/j.enbuild.2011.12.037.

[17] F. Jazizadeh, W. Jung, Personalized thermal comfort inference using RGB video images for distributed HVAC control, Applied Energy. 2018. vol. 220, 829-841. doi:10.1016/j.apenergy.2018.02.049.

[18] R. Melfi, B. Rosenblum, B. Nordman, K. Christensen, Measuring 
building occupancy using existing network infrastructure, in: International Green Computing Conference, 2011.

[19] M. Kapsalaki, V. Leal, Recent progress on net zero energy buildings, Advances in Building Energy Research. 2011. vol. 5, 129-162. doi:10.1080/17512549.2011.582352.

[20] I. Sartori, A. Napolitano, K. Voss, Net zero energy buildings: a consistent definition framework, Energy and Buildings. 2012. vol. 48, 220-232. doi:10.1016/j.enbuild.2012.01.032.

[21] S. Pigg, M. Eilers, J. Reed, Behavioral Aspects of Lighting and Occupancy Sensors in Privates Offices: A case study of a University Office Building, in: ACEEE 1996 Summer Study on Energy Efficiency in Buildings, 1996: pp. 161-170.

[22] B. Dong, M.B. Kjærgaard, M. De Simone, H.B. Gunay, W. O'brien, D. Mora, J. Dziedzic, J. Zhao, Sensing and Data Acquisition, in: A Wagner, W. O'brien, B. Dong (Eds.), Exploring Occupant Behavior in Buildings- Methods and Challenges, Springer International Publishing AG, 2018. doi:10.1007/978-3-319-61464-9.

[23] D. Liu, X. Guan, Y. Du, Q. Zhao, Measuring indoor occupancy in intelligent buildings using the fusion of vision sensors, Measurement Science and Technology. 2013. vol. 24, 074023. doi:10.1088/09570233/24/7/074023

[24] D. Mora, G. Fajilla, M.C. Austin, M. De Simone, Occupancy patterns obtained by heuristic approaches: cluster analysis and logical flowcharts. A case study in a university office, Energy and Buildings. 2019. doi:10.1016/j.enbuild.2019.01.023.
[25] B. Dong, K.P. Lam, Building energy and comfort management through occupant behaviour pattern detection based on a large-scale environmental sensor network, Journal of Building Performance $\begin{array}{llll}\text { Simulation. } 2011 . & \text { vol. } & 4, & 359-369 .\end{array}$ doi:10.1080/19401493.2011.577810.

[26] A. Ebadat, G. Bottegal, D. Varagnolo, B. Wahlberg, K.H. Johansson, Estimation of building occupancy levels through environmental signals deconvolution, in: Proceedings of the 5th ACM Workshop on Embedded Systems For Energy-Efficient Buildings - BuildSys'13, 2013: pp. 1-8. doi:10.1145/2528282.2528290.

[27] J. Yang, M. Santamouris, S.E. Lee, Review of occupancy sensing systems and occupancy modeling methodologies for the application in institutional buildings, Energy and Buildings. 2015. vol. 121 1-6. doi:10.1016/j.enbuild.2015.12.019.

[28] C. Carpino, D. Mora, M. De Simone, On the use of questionnaire in residential buildings. A review of collected data, methodologies and objectives, Energy and Buildings. 2019. doi:10.1016/j.enbuild.2018.12.021.

[29] W. Wang, J. Chen, T. Hong, Occupancy prediction through machine learning and data fusion of environmental sensing and Wi-Fi sensing in buildings, Automation in Construction. 2018. vol. 94, 233-243. doi:10.1016/j.autcon.2018.07.007.

[30] Z. Chen, C. Jiang, L. Xie, Building occupancy estimation and detection: A review, Energy and Buildings. 2018. vol. 169, 260-270. doi:10.1016/j.enbuild.2018.03.084. 